\title{
Treatment decision in asymptomatic aortic valve stenosis: role of exercise testing
}

\author{
M C M Amato, P J Moffa, K E Werner, J A F Ramires
}

\begin{abstract}
Objective-To determine the prognostic value of exercise testing, valve area, and maximum transaortic pressure gradient in asymptomatic patients with aortic valve stenosis.

Setting-The outpatient service of a tertiary referral centre for cardiology.

Design-Prospective clinical study.

Patients-66 consecutive patients with isolated severe aortic stenosis (aortic valve area $\leqslant 1.0 \mathrm{~cm}^{2}$ ) were selected over a 58 month period. Mean (SD) follow up was 14.77 (11.93) months.

Interventions-At the initial visit Doppler echocardiography and exercise testing were performed to evaluate ST segment depression and the development of symptoms of aortic stenosis, ventricular arrhythmia, or inadequate rise of systolic blood pressure during exercise. Follow up clinical examinations were performed every three months thereafter to record the onset of symptoms.

Main outcome measures-Sudden death or the development of symptoms.

Results-Eight patients developed dizziness during exercise testing but made a rapid and spontaneous recovery. No other complications of exercise testing occurred. Survival curves, with or without the occurrence of end point events for the variables studied, showed significant differences for positive versus negative exercise testing $(\mathrm{p}=0.0001)$ and aortic valve area $<0.7 \mathrm{~cm}^{2} v \geqslant 0.7 \mathrm{~cm}^{2}(\mathrm{p}=0.0021)$. There was no relation between the end points and transaortic gradient $(p=0.6882)$. In multivariate analysis, a hazard ratio of 7.43 was calculated for patients with a positive versus a negative exercise stress test. Although asymptomatic in daily life, $6 \%$ of the patients $(4 / 66)$ experienced sudden death; all these had a positive exercise test and an aortic valve area of $\leqslant 0.6 \mathrm{~cm}^{2}$.
\end{abstract}

Conclusions-Exercise testing is safe and is of prognostic value in asymptomatic patients with aortic stenosis.

(Heart 2001;86:381-386)

Keywords: aortic valve stenosis; exercise testing; Doppler echocardiography

Studies investigating the natural history of aortic valve stenosis in adults show that the disease is progressive, but the longer we can preserve a patient's natural valve, the lower the risk of future complications. ${ }^{1-3}$ As stenosis increases, compensatory mechanisms fail and the four major symptoms - dyspnoea, angina, syncope, and arrhythmias - start to occur; other common symptoms include dizziness, weakness, fatigue, and exercise intolerance. ${ }^{45}$

The risk of sudden death in asymptomatic patients appears to be less than $1 \%{ }^{6}$ but it increases once symptoms appear. ${ }^{23-10}$ Thus the development of the classic symptoms is seen as a turning point in the patient's disease, and the natural valve is replaced. At present, surgery in asymptomatic patients is not recommended in our service. Significant long term complications from mechanical and bioprosthetic valves occur at a rate of at least $2-3 \%$ a year, and there is a $1 \%$ annual incidence of death caused directly by the prosthesis. ${ }^{11-13}$ Thus even if surgical mortality can be minimised the combined risks of surgery and the late complications of a prosthesis exceed the risk of sudden death in many asymptomatic patients. Despite these considerations, there are still differences of opinion among clinicians over the indications for corrective surgery in such patients.

In some patients the clinical presentation of symptoms is clear, while in others they may be disguised by the sedentary life that is recommended for patients with this disease. The patient might also underestimate the symptoms-for example, occasional dizziness - and therefore not report them to the cardiologist. Furthermore, chest pain is often atypical. Thus the question of whether a particular patient is really asymptomatic arises commonly.

Until recently, it has been considered that stress testing is contraindicated in patients with severe aortic stenosis, mainly for safety reasons. ${ }^{6}$ Thus few studies involving exercise have been performed. Our aim in this study was to expose patients with severe aortic stenosis to the potential risk of exercise in a safe clinical environment. We compared the prognosis of patients with a positive exercise test (development of precordial chest pain, syncope, or dizziness; abnormal haemodynamic response; ECG changes) with that of patients with a negative test. We also investigated the prognostic value of the aortic valve area and the maximum transaortic pressure gradient.

\section{Methods}

PATIENT CHARACTERISTICS

On the basis of the selection criteria described below, we followed up 66 of a total of 853 patients diagnosed with severe aortic stenosis at the time of their first visit to our clinic. The age range of the cohort was $18-80$ years; their 
mean (SD) age was 49.5 (14.9) years. Forty four $(66.7 \%)$ of the patients were men and 22 $(33.3 \%)$ women. Aortic valve area ranged from $0.3-1.0 \mathrm{~cm}^{2}$.

\section{PATIENT SELECTION}

In all, 853 consecutive patients attending our outpatient valve disease service between February 1987 and February 1992 were diagnosed as having aortic valve stenosis. The 66 patients selected from this group for inclusion in our cohort were chosen on the following five criteria:

- the absence of symptoms characteristic of aortic valve disease-dyspnoea, angina pectoris, syncope, arrhythmias, and a range of minor symptoms (dizziness, weakness, fatigue, exercise intolerance)-as well as symptoms of other chronic conditions, to ensure that the patients were in the latent period characteristic of stenotic aortic disease;

- severe aortic stenosis with aortic valve area $\leqslant 1 \mathrm{~cm}^{2}$, without coexisting valve disease; the value of $\leqslant 1 \mathrm{~cm}^{2}$ was selected because haemodynamic alterations start to occur at this level of stenosis, which is therefore classified as severe ${ }^{9}$;

- absence of arrhythmia, left bundle branch block, or ST-T segment depression, as determined by ECG evaluation, confirming that any ECG changes during the exercise test were interpretable;

- absence of coronary artery disease or other heart disease, as determined by cardiac catheterisation performed no longer than six months before entrance into the protocol, to exclude the possibility of a coronary origin of the symptoms common to both diseases, such as precordial chest pain;

- absence of comorbid disease associated with symptoms that could interfere with clinical evaluation and preclude the performance of an exercise test.

All patients were informed about the procedures, benefits, and risks involved in participating in the study protocol and gave their informed consent, which was approved by the review and ethics commission of the hospital.

PATIENT EVALUATION AND FOLLOW UP

Physical examination and a detailed interview to detect the development of symptoms typical of aortic stenosis - such as precordial chest pain, signs of heart failure, dizziness, or syncope-were performed in the selected patients every three months during the study period (range of follow up 2.62-57.6 months). Doppler echocardiography was performed at the initial visit to determine the valve area and mean and maximum transaortic pressure gradient. Electrocardiography and exercise testing were also performed at the initial visit.

We defined two end point events for the study - the appearance of symptoms in daily life, and sudden death. At the time of the study, our hospital's management strategy for aortic stenosis did not include exercise testing in the routine evaluation of patients with aortic stenosis.
Doppler echocardiography

All patients underwent Doppler and cross sectional echocardiography while at rest and lying in the left lateral decubitus position. Echocardiography was performed using either an Aloka SSD 725 and 860 (Aloka, Tokyo, Japan) with mechanical 2.0 and $3.0 \mathrm{MHz}$ transducers or an ATL Ultramark 9 HDI system (ATL (Philips Medical Systems), Bothell, Washington, USA), with electronic 2.5 and $3.5 \mathrm{MHz}$ transducers. The different equipment in use had no influence on the methodology or the accuracy of the results. On the parasternal long axis view, ventricular outflow tract diameter was measured at mid-systole, just proximal to the insertion of the aortic leaflet into the annulus of the valve. The maximum aortic jet velocity was recorded using continuous wave Doppler in the long axis view to identify the highest velocity signal. Left ventricular outflow tract velocity was recorded from the apical angle using pulsed Doppler. Maximum and mean transaortic pressure gradients were calculated using the Bernoulli equation. Aortic valve area was calculated from the continuity equation. ${ }^{14}{ }^{15}$

\section{Exercise testing}

Exercise testing was performed using a treadmill with a mobile ramp. As is the established routine in our clinic, the Ellestad protocol was applied $^{16}$ (appendix). Testing took place in a specially equipped laboratory in the presence of two physicians so that complications could be managed effectively. The age related peak heart rate was determined using the formula $(210$ - age). Submaximal frequency corresponded to $85 \%$ of this value.

A 12 lead ECG, heart rate, and systolic and diastolic blood pressure were recorded with the patients in the standing position at rest and then after two minutes of each stage of the exercise protocol and at peak exercise. A three lead ECG was monitored continuously. These variables were also recorded every two minutes after exercise for at least six minutes, or until the ST segment returned to baseline, blood pressure recovered, and symptoms disappeared. Exercise was interrupted promptly when the test was considered positive or when the patient reached the age related maximum heart rate. The need to interrupt the test was determined according to the criteria of the American Heart Association. ${ }^{17}$

\section{TEST INTERPRETATION}

The exercise test was considered positive if there was a horizontal or downsloping ST segment depression of $\geqslant 1 \mathrm{~mm}$ in men or $\geqslant 2 \mathrm{~mm}$ in women, or an upsloping ST segment depression of $\geqslant 3 \mathrm{~mm}$ in men, measured 0.08 seconds after the $\mathrm{J}$ point. To make the test more specific, upsloping ST segment depression in women was considered negative. The exercise test was also considered positive if symptoms of aortic stenosis occurred (precordial chest pain or near syncope), if the ECG showed a complex ventricular arrhythmia, or if systolic blood pressure failed to rise 
Table 1 Patient characteristics according to the appearance of end point events

\begin{tabular}{lccll}
\hline & Mean & SD & End point & $n$ \\
\hline Age (years) & 44.2 & 13.7 & No & 28 \\
& 53.7 & 14.7 & Yes & 38 \\
Aortic valve area $\left(\mathrm{cm}^{2}\right)$ & 0.72 & 0.16 & No & 28 \\
Transaortic pressure gradient $(\mathrm{mm} \mathrm{Hg})$ & 0.54 & 0.14 & Yes & 38 \\
& 73.3 & 25.3 & No & 27 \\
ST segment depression $(\mathrm{mm})^{\star}$ & 90.3 & 36.3 & Yes & 38 \\
p $(\mathrm{mm} \mathrm{Hg})$ & 0.77 & 1.17 & No & 26 \\
& 1.87 & 1.75 & Yes & 38 \\
Follow up (months) & 35.6 & 15.1 & No & 26 \\
& 20.1 & 17.5 & Yes & 38 \\
& 23.6 & 12.5 & No & 28 \\
& 8.3 & 5.9 & Yes & 38
\end{tabular}

${ }^{\star} \mathrm{ST}$ segment depression 0.08 seconds after J point according to defined criteria. End point, symptoms or death; $\Delta \mathrm{p}$, rise of systolic blood pressure from baseline.

Table 2 Exercise testing results in the entire cohort of patients $(n=66)$

\begin{tabular}{lrr}
\hline & \multicolumn{1}{l}{$n$} \\
\hline & \multicolumn{1}{c}{$\%$} \\
\hline Negative & 22 & 33.3 \\
Positive & 44 & 66.7 \\
$\quad$ Symptoms ${ }^{\star}$ (3 arrhythmias) & 7 & 15.91 \\
Y point & 8 & 18.18 \\
$\Delta \mathrm{p}$ & 4 & 9.09 \\
Symptoms $+\Delta \mathrm{p}$ & 10 & 22.73 \\
Symptoms + Y point & 3 & 6.82 \\
Y point $+\Delta \mathrm{p}$ & 9 & 20.45 \\
Symptoms + Y point $+\Delta \mathrm{p}$ & 3 & 6.82
\end{tabular}

^Dizziness, precordial chest pain, and arrhythmias; $\Delta \mathrm{p}$, change in systolic blood pressure from baseline; Y point, $0.08 \mathrm{~s}$ after $\mathrm{J}$ point in the ST segment depression.

by $\geqslant 20 \mathrm{~mm} \mathrm{Hg}$ during exercise compared with baseline.

\section{STATISTICAL ANALYSIS}

Initially, a descriptive analysis of the data was performed, evaluating age, aortic valve area, maximum transaortic pressure gradient, $\mathrm{ST}$ segment depression during exercise test 0.08 seconds after the J point, difference in systolic blood pressure before and at peak exercise, and follow up time. The data were then analysed separately for the patients who reached one of the end points of the study, as defined above, and the patients who survived and remained asymptomatic until the study was completed.

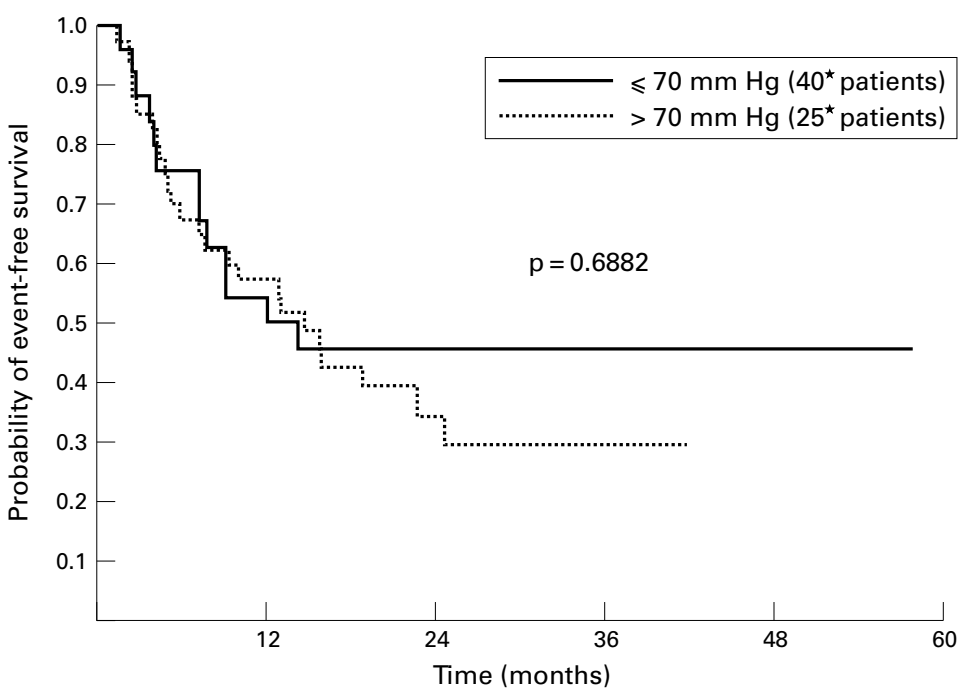

* One patient did not have his valvar gradient measured

Figure 1 Kaplan-Meier life table analysis for probability of event-free survival over 60 months for patients with asymptomatic severe aortic stenosis, according to maximum transaortic pressure gradient $\leqslant 70 \mathrm{~mm} \mathrm{Hg}$ or $>70 \mathrm{~mm} \mathrm{Hg}$.
To establish borderlines for decision making, patients were divided into two subgroups for each of the following five variables: positive and negative exercise test results; aortic valve area $<0.7 \mathrm{~cm}^{2}$ and $\geqslant 0.7 \mathrm{~cm}^{2}$; peak gradient $\leqslant 70 \mathrm{~mm} \mathrm{Hg}$ and $>70 \mathrm{~mm} \mathrm{Hg} ;<50$ and $\geqslant 50$ years of age; and male and female. Kaplan-Meier life table analysis was used to calculate actuarial survival curves for the variables studied. ${ }^{18}$ Actuarial curves were compared using the log-rank method.

The variables that showed significant differences in survival were included in a Cox proportional hazards regression analysis to determine the ratio of risk of the end point event for patients with or without the covariate.

\section{Results}

PATIENT CHARACTERISTICS

The 66 patients selected according to the inclusion criteria had a mean (SD) age of 49.7 (14.9) years. The group consisted of 22 women $(33.3 \%)$ and 44 men $(66.7 \%)$. Mean aortic valve area was $0.61(0.17) \mathrm{cm}^{2}$, with a minimum of $0.30 \mathrm{~cm}^{2}$. The average maximum transaortic pressure gradient was 83.3 (33.0) $\mathrm{mm} \mathrm{Hg}$.

Patients who remained asymptomatic had a mean follow up time of 23.6 (12.5) months. Among the patients who developed symptoms, the mean follow up was 8.3 (5.9) months. Generally, patients who survived symptom-free were younger, had a larger aortic valve area, and had a lower peak gradient (table 1 ).

EXERCISE TESTING

Exercise testing was positive in 44 of the 66 patients $(66.7 \%)$ (table 2$)$. Table 2 shows the characteristics of the positive exercise tests in our group of patients. Thirty five patients with a positive exercise test reached an end point, compared with only three patients with a negative test $(p=0.001)$.

Among the group of 66 patients, 20 developed either dizziness $(35 \% ; 7 / 20)$, precordial chest pain $(60 \% ; 12 / 20)$, or both $(5 \%$; $1 / 20$ ) during exercise testing. All 20 patients recovered within 10 minutes without needing treatment. However, all those who experienced dizziness or precordial chest pain during the test developed spontaneous symptoms later.

Three $(6.8 \%)$ of the 44 patients who had a positive stress test were identified as having a ventricular arrhythmia. One of these patients reached an end point at 5.44 months and two remained asymptomatic throughout the mean 21.9 months follow up (table 2).

EVENT-FREE SURVIVAL

We then used Kaplan-Meier life table analysis to calculate the actuarial event-free survival curves over the 60 month observation period. The probability of event-free survival (with SEM) was $0.71(0.06)$ at six months; 0.57 (0.06) at 12 months; $0.43(0.06)$ at 18 months; and $0.38(0.06)$ at 24 months. There was no difference in event-free survival between men and women. There was a slightly higher probability for staying event-free in patients younger than 50 years $(\mathrm{p}=0.0384)$. 


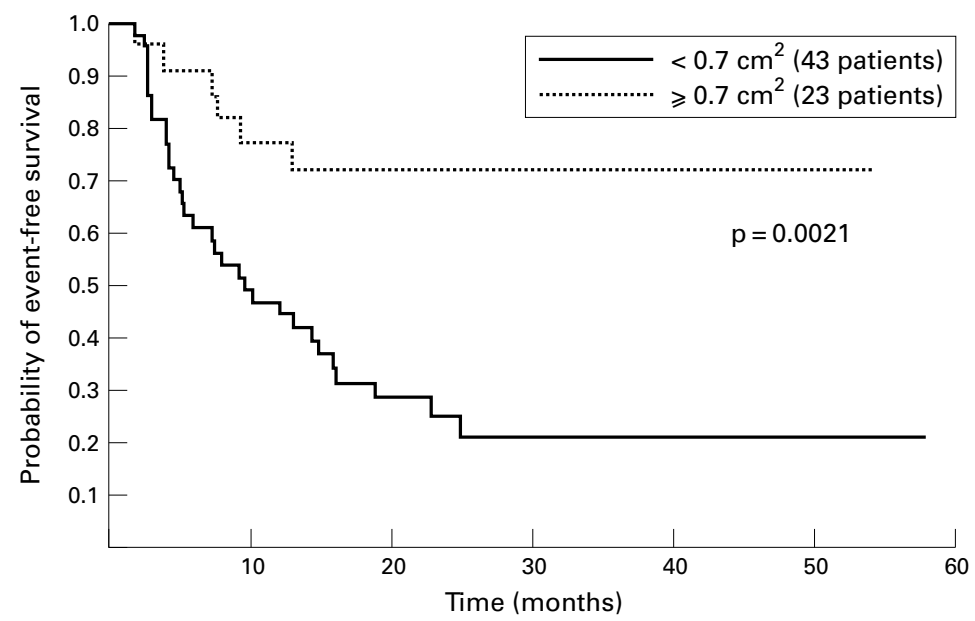

Figure 2 Kaplan-Meier life table analysis for probability of event-free survival over 60 months for patients with asymptomatic severe aortic stenosis, according to aortic valve area $<0.7 \mathrm{~cm}^{2}$ or $\geqslant 0.7 \mathrm{~cm}^{2}$.

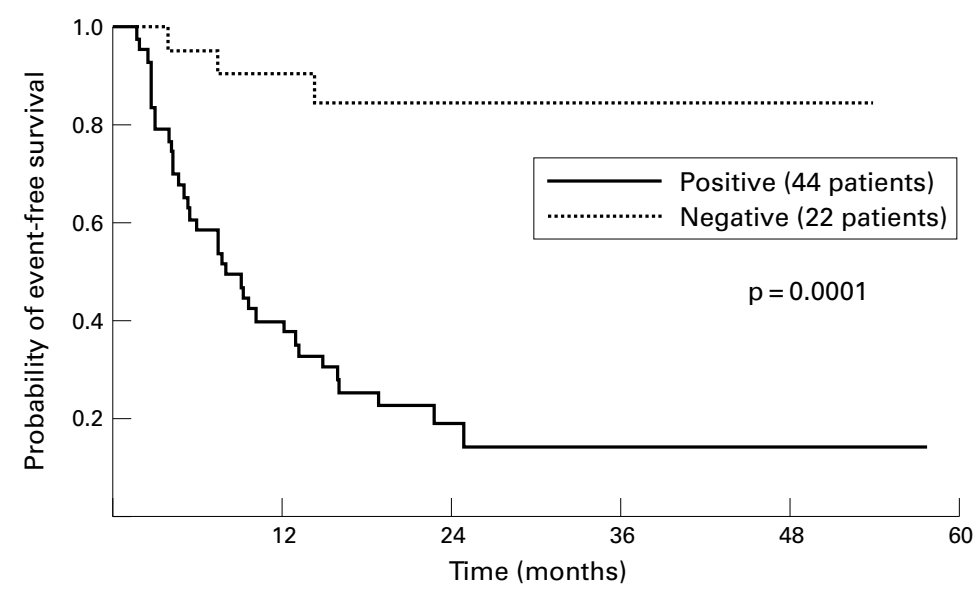

Figure 3 Kaplan-Meier life table analysis for probability of event-free survival over 60 months for patients with asymptomatic severe aortic stenosis, according to positive or negative results of exercise testing.

Transvalvar aortic pressure gradient

For the maximum transvalvar aortic pressure gradient, patients were divided in those with a gradient of $\leqslant 70 \mathrm{~mm} \mathrm{Hg}$ or $>70 \mathrm{~mm} \mathrm{Hg}$, using the cut off point suggested by Horstkotte and Loogen. ${ }^{3}$ There was no significant difference in event-free survival between these subgroups $(p=0.6882)$ (fig 1$)$.

\section{Aortic valve area}

Analysis of the aortic valve area, using the value $<0.7 \mathrm{~cm}^{2}$ or $\geqslant 0.7 \mathrm{~cm}^{2}$, showed that there was a significantly better chance of event-free survival for the patients with a valve area $\geqslant 0.7 \mathrm{~cm}^{2}(\mathrm{p}=0.0021)$ (fig 2$)$.

Table 3 Characteristics of patients who experienced sudden death $(n=4)$

\begin{tabular}{|c|c|c|c|c|c|c|c|}
\hline \multirow[b]{2}{*}{$\operatorname{Sex}$} & \multirow{2}{*}{$\begin{array}{l}\text { Age } \\
\text { (years) }\end{array}$} & \multirow{2}{*}{$\begin{array}{l}\text { Valve area } \\
\left(\mathrm{cm}^{2}\right)\end{array}$} & \multirow{2}{*}{$\begin{array}{l}\text { Gradient } \\
(m m \mathrm{Hg})\end{array}$} & \multicolumn{3}{|c|}{ Exercise testing } & \multirow{2}{*}{$\begin{array}{l}\text { Follow up } \\
\text { ( } \triangle \text { days) }\end{array}$} \\
\hline & & & & Symptom & $S T(\mathrm{~mm})$ & $\Delta p$ & \\
\hline Male & 41 & 0.38 & 110 & Angina & 1.5 & 10 & 130 \\
\hline Female & 59 & 0.60 & 80 & Angina & - & 0 & 482 \\
\hline Male & 49 & 0.49 & 136 & - & 3.5 & 10 & 180 \\
\hline Male & 48 & 0.48 & 99 & Angina & 2.0 & 0 & 140 \\
\hline
\end{tabular}

$\Delta \mathrm{p}$, change in systolic blood pressure from baseline in $\mathrm{mm} \mathrm{Hg}$; ST, ST segment depression 0.08 seconds after J point.

\section{Exercise testing}

The most significant correlation was between the result of exercise testing and survival ( $p=0.0001$ ) (fig 3). Of the 38 patients who reached one of the end points of the study, 35 $(92.1 \%)$ had a positive stress test and only three $(7.9 \%)$ had a negative test. After 24 months, the probability of a patient with a positive test surviving event-free was only 0.19 , compared with 0.85 in those with a negative test (fig 3).

\section{RISK RATIO}

We included variables that showed significance (age, aortic valve area, and exercise testing) in a multivariate analysis. We were able to show that the estimated risk for a patient with a positive exercise test for any reason-that is, ST segment depression, symptoms, or inadequate haemodynamic response-was 7.6 (0.6) times higher than for a patient with a negative test for developing an end point. The risk ratio for valve area was 1.48 and for age, 1.16.

Four of the 66 patients $(6.1 \%)$ experienced sudden death. All had valve areas of $\leqslant 0.6 \mathrm{~cm}^{2}$, a high maximum transaortic pressure gradient of more than $80 \mathrm{~mm} \mathrm{Hg}$, and a positive stress test (table 3).

\section{Discussion}

The overall actuarial survival curve of the 66 patients in our cohort showed that only $50 \%$ of the patients were symptom-free 16 months after the initial diagnosis. This illustrates the rapid rate of progression of the degenerative calcific process and the high morbidity and mortality in patients with severe aortic stenosis.

Some degree of aortic regurgitation is involved in about $80 \%$ of patients with aortic stenosis. Coexisting mitral valve disease, in particular regurgitation, is also common. ${ }^{15}$ Our cohort consisted of 66 patients with pure aortic stenosis. This selection was possible because of the large numbers of patients in our outpatient clinic. Our aim was to exclude the influence of other factors in the progression of the disease. Among our study group, $6.1 \%$ experienced sudden death, which represents an annual rate of $1.2 \%$. In published reports, the annual rate of sudden death in asymptomatic patients is estimated to be less than $1 \% .^{361920}$ We attributed the deaths in our cohort to aortic valve stenosis because coronary artery disease and other important systemic diseases were excluded and death occurred suddenly. The patients who died were asymptomatic in daily life. However, in addition to having an aortic valve area of $\leqslant 0.60 \mathrm{~cm}^{2}$, they also had a positive exercise test result. Three had precordial chest pain during exercise and one had significant ST segment depression $(3.5 \mathrm{~mm})$. None of these patients was able to raise their blood pressure by more than $10 \mathrm{~mm} \mathrm{Hg}$ during stress testing. We emphasise that these four patients remained asymptomatic until death occurred, and therefore they were never referred for valve replacement.

In a 1997 study, Otto and colleagues showed that the development of symptoms is related to the peak gradient. ${ }^{14}$ These investigators found a 
substantial overlap of pressure gradients between symptomatic and asymptomatic patients. However, the transvalvar gradient was not related to event-free survival in the analysis of our cohort. This emphasises that severe aortic stenosis cannot be defined by a single value. The maximum transaortic pressure gradient varies with myocardial function and peripheral resistance. In patients with a depressed myocardium, the pressure gradient may be small despite a severely reduced valve area. ${ }^{21}$

The close association between an aortic valve area of $<0.7 \mathrm{~cm}^{2}$ and a larger number of events $(84.2 \%)$ in our group shows that the degree of stenosis alone is an important index of prognosis. Nevertheless, there is an association between valve area and the appearance of symptoms in such patients. ${ }^{14}$ It is remarkable that, at the time of inclusion in our protocol, 43 patients had a valve area of $<0.7 \mathrm{~cm}^{2}$ and were still asymptomatic. Ten of these patients remained asymptomatic, while six others with valve areas $\geqslant 0.7 \mathrm{~cm}^{2}$ (of a total of 23 patients) developed end point symptoms.

The importance given to valve area derives from the theory that the risk increases when the area falls below a specific numerical value. ${ }^{14}$ However, there is support for a more dynamic concept, that symptom onset in the individual patient depends on the severity of the aortic stenosis as well as on left ventricular function and the status of the peripheral circulation. ${ }^{2-5} 15$ Symptoms occur when the peripheral demands exceed the cardiac output. The degree of valve stenosis at which this happens varies from subject to subject. Because exercise provokes greater peripheral demand, symptoms should become manifest under such circumstances. Our prospective study showed that those patients who manifest symptoms or ECG changes during exercise testing are more likely to experience a clinical end point.

In a 1979 study using cineangiography of the aortic root performed at rest and during exercise, Richardson and colleagues suggested there was a dynamic component to aortic valve obstruction, after noting that the calculated area during exercise was larger in some patients than in others. ${ }^{22}$ In a later study, Otto and associates showed that Doppler echocardiography made it possible to assess haemodynamic variables during exercise in patients with aortic stenosis. ${ }^{23}$ Cardiac output rose appropriately with exercise, owing entirely to an increase in heart rate, whereas the mean stroke volume fell slightly. The maximum and mean transaortic pressure gradient increased with exercise. There was no significant change in the valve area. Nevertheless, Otto reported a trend towards increasing valve area with exercise in some patients. In a further prospective study, Otto found that there was a greater increase in valve area with exercise in patients who remained asymptomatic than in those with a clinical end point. ${ }^{24} \mathrm{He}$ suggested that this phenomenon may reflect greater leaflet stiffness in subjects with more severe disease. These data demonstrate how individual differences contribute to the prognosis of severe aortic stenosis. Further, the data may explain why the subjects with a negative exercise test in our study had a better prognosis.

Minor complications during exercise testing in asymptomatic patients with aortic valve stenosis have been few, with reports of a fall in blood pressure by $>10 \mathrm{~mm} \mathrm{Hg}$ in $9 \%$ of patients, and ST depression of $>2 \mathrm{~mm}$ in $2 \%$ of patients. ${ }^{14}$ In our study, no such fall in systolic blood pressure occurred, whereas 30\% (20/ 66) had ST depression of $>2 \mathrm{~mm}$. Otto and associates stated that angina occurred in 3\% of their subjects, dizziness in $1 \%$, and arrhythmias in $1 \%$. In our cohort, $19.7 \%(n=13)$ experienced angina, $10.6 \%(\mathrm{n}=7)$ dizziness, and $4.5 \%(n=3)$ arrhythmia. The higher rate of complications in our study may reflect differences in the methods. In the cited study ${ }^{14}$ several exercise tests were performed in the same subject, whereas in our study we performed only one test per patient. The previous study also included patients with less severe disease. The mean aortic gradient at baseline was 29 (11) $\mathrm{mm} \mathrm{Hg}$, and the aortic valve area was $1.3(0.5) \mathrm{cm}^{2},{ }^{19}$ compared with 83.3 (33.0) $\mathrm{mm} \mathrm{Hg}$ and $0.61(0.17) \mathrm{cm}^{2}$, respectively, in our study. Nevertheless, there were no major complications in our cohort, and examinations were interrupted promptly when minor complications occurred.

Exercise testing has previously been shown to be a predictor of clinical outcome. ${ }^{1425}$ Because the indications for surgery are still based on the development of symptoms, our data suggest that the occurrence of symptoms or of ST depression during exercise testing should be considered an indication for valve replacement. In the actuarial survival curve, patients with a positive exercise testing result had a statistically slimmer chance of event free survival. Multivariate analysis showed that subjects with a positive exercise testing had a 7.64-fold risk of experiencing one of the end point events.

Precordial chest pain, which occurs as a result of myocardial oxygen supply-demand imbalance, first manifests itself during exercise. ${ }^{26}$ Dizziness or syncope could be caused by an inappropriate ventricular baroreceptor response leading to peripheral vasodilatation and consequently to hypotension, ${ }^{27}$ or to an inability to increase cardiac output across a stenotic valve on exertion. ${ }^{24}$ An inadequate rise in systolic blood pressure is caused by a limited capacity to increase cardiac output. These symptoms also appear during exercise testing. Three of the four patients who died in our cohort experienced precordial chest pain during stress testing. All the patients with symptoms during exercise testing developed end point symptoms, and the event-free survival of subjects with a negative stress test was significantly longer than for patients with a positive test. These results suggest that asymptomatic patients should be submitted to exercise testing to evaluate their functional status. While it has been suggested that one should consider only an inadequate increase in systolic blood pressure during exercise as a predictive variable, our data suggest that a positive exercise test, for any reason, is an independent predictor of outcome. 
Table 4 Standart Ellestad protocol ${ }^{16}$

\begin{tabular}{llll}
\hline Stage number & Speed (mile/h) & Grade (\%) & Time (min) \\
\hline 1 & 1.7 & 10.0 & 3.0 \\
2 & 3.0 & 10.0 & 2.0 \\
3 & 4.0 & 10.0 & 2.0 \\
4 & 5.0 & 10.0 & 2.0 \\
5 & 5.0 & 15.0 & 2.0 \\
6 & 6.0 & 15.0 & 2.0 \\
7 & 7.0 & 15.0 & 2.0 \\
8 & 3.0 & 5.0 & 2.0 \\
9 & 1.7 & 2.5 & 2.0 \\
10 & 1.0 & 0.0 & 0.0 \\
11 & - & - & - \\
12 & - & - & - \\
13 & - & - & - \\
14 & - & - & - \\
15 & - & - & - \\
16 & - & - & - \\
\hline
\end{tabular}

\section{CONCLUSIONS}

Exercise testing contributes valuable information to the evaluation of the patient with aortic valve stenosis. Together with the measurement of valve area by Doppler echocardiography, it may assist in the often difficult decision about whether to recommend aortic valve replacement.

\section{Appendix}

ELLESTAD PROTOCOL

Ellestad was the protocol used (table 4). This was modified to evaluate the patients' stress tolerance. The objective was to reach the patients' maximum heart rate, but exercise was stopped when any symptom occurred. A two minute warm-up stage was added before the protocol started; in this, the exercise was performed with the treadmill level and with a velocity similar to that of the first test stage. Where a patient could not achieve the second test stage, we controlled the speed manually, modifying the protocol on an individual basis.

1 Freeman WK, Hartzell HV, Schaff HV, et al. Ultrasonic aortic valve decalcification: serial Doppler echocardiographic follow-up. F Am Coll Cardiol 1990;16:623-30.

2 Kelly TA, Rothbart RM, Cooper M, et al. Comparison of outcome of asymptomatic to symptomatic patients older than 20 years of age with valvular aortic stenosis. $A m \mathcal{F}$ Cardiol 1988;61:123-30.

3 Horstkotte DD, Loogen F. The natural history of aortic valve stenosis. Eur Heart f 1988;9:57-64.

4 Turina J, Hess D, Sepulcri F, et al. Spontaneous course of Tortic valve disease. Eur Heart f 1987:8:471-83.

5 Pellikka PA, Nishimura RA, Bailey KR, et al. The natural history of adults with asymptomatic, hemodynamically significant aortic stenosis. F Am Coll Cardiol 1990;15:101217.
6 Bonow RO, Carabello B, deLeon AC, et al. ACC/AHA guidelines for the management of patients with valvular heart disease: a report of the American College of Cardiology/American Heart Association task force on practice guidelines (committee on management of patients with valvular heart disease). 7 Am Coll Cardiol 1998;32: 1486-588.

7 Olesen $\mathrm{KH}$, Warburg E. Isolated aortic stenosis: the late prognosis. Acta Med Scand 1958;160:437-46.

8 Frank S, Johnson A, Ross JJ. Natural history of valvular aortic stenosis. Br Heart $\mathcal{F}$ 1973;35:41-6.

9 Nitta M, Nakamura T, Hultgren HN, et al. Progression of aortic stenosis in adult men. Chest 1987;92:40-3.

10 Rapaport E. Natural history of aortic and mitral valve disease. Am f Cardiol 1975;35:221-7.

11 Vongpatanasin W, Hillis LD, Lange RA. Prosthetic heart valves. $N$ Engl F Med 1996;335:407-16.

12 Amato MCM, Pomeratzeff F, Grinberg M, et al. [Immediate and long-term prognosis of patients with porcine bioprosthesis] Arq Bras Cardiol 1988;51:381-4.

13 Amato MCM, Pomerantzeff F, Grinberg M, et al. [Implant of Starr-Edwards mechanical prosthesis for more than 15 years: analysis of 34 cases.] Arq Bras Cardiol 1987;48:299301.

14 Otto CM, Burwash IG, Legget ME, et al. Prospective study of the asymptomatic valvular aortic stenosis: clinical, echocardiographic and exercise predictors of outcome. Circulation 1997;95:2262-70.

15 Archer SI, Mike DK, Hetland MB, et al. Usefulness of mean aortic valve gradient and left ventricular diastolic filling pattern for distinguishing the symptomatic from the asymptomatic patients. Am f Cardiol. 1994;73:275-81.

16 Ellestad MH. Stress testing: principles and practice. Philadelphia: Davis, 1975.

17 Gibbsons RJ, Balady GJ, Beasley JW, et al. ACC/AHA guidelines for exercise testing: a report of the American College of Cardiology/American Heart Association task force on practice guidelines (committee on exercise force on practice guidelines (committee
testing). F Am Coll Cardiol 1997;30:260-315.

18 Lee EP. Statistical methods for survival data analysis. Belmont: Life Time Learning Publication, 1980:75-154

19 Ross J, Braunwald E. Aortic stenosis. Circulation 1968;38: $61-7$.

20 Iivanainen $\mathrm{AM}$, Lindroos $\mathrm{M}$, Tilvis $\mathrm{R}$, et al. Natural history of aortic valve stenosis of varying severity in the elderly. $\mathrm{Am}$ of aortic valve stenosis of vary

21 Braunwald E. On the natural history of severe aortic stenosis. F Am Coll Cardiol 1990;15:1018-20.

22 Richardson JW, Anderson FL, Tsagaris TJ. Rest and exercise hemodynamic study in patients with isolated aortic stenosis. Cardiology 1979;64:1-11.

23 Otto CM, Pearlman AS, Kraft CD, et al. Physiologic changes with maximal exercise in asymptomatic valvular aortic stenosis assessed by Doppler Echocardiography. $\mathcal{F}$ Am Coll Cardiol 1992;20:1160-7.

24 Otto CM. Aortic stenosis. Clinical evaluation and optimal timing for surgery. Cardiol Clin 1998;16:353-73.

25 Amato MCM. [Asymptomatic aortic stenosis: evaluation through exercise testing]. Acta Cirurgica Brasillira 1995;10: $77-83$.

26 Gould KL. Why angina pectoris in aortic stenosis? Circulation 1997;59:790-2.

27 Johnson AM. Aortic stenosis, sudden death, and the left ventricular baroreceptors. Br Heart $\mathcal{F}$ 1971;33:1-5.

28 Grech ED, Ramsdale DR. Exertional syncope in aortic stenosis: evidence to support inappropriate left ventricular response. Am Heart F 1991;121:603-6. 